myxoma arising by a pedicle from the anterior wall of the right ventricle, located inside the pericardium, has previously been reported.

After total excision of the mass, microscopic examination revealed that the tumor had the characteristics of a myxoma covered by epicardial cells. The histogenesis of cardiac myxoma is uncertain. ${ }^{6}$ The immunohistochemical profile of this mass revealed endothelial antigens, vimentin, and smooth muscle-actin positive cells, suggesting a neoplastic origin of the myxoma from endothelial and mesenchymal cells. These multipotential cells in a subepicardial location could differentiate into several cell types, including endothelial cells, fibrocytes, and smooth muscle cells.

\section{REFERENCES}

1. Chitwood WR Jr. Cardiac neoplasm: current diagnosis, pathology, and therapy. J Card Surg 1988;3:119-54.

2. Wold LE, Lie JT. Cardiac myxoma: a clinicopathologic profile. Am J Pathol 1980;101:219-33.

3. Farah MG. Familial cardiac myxoma: a study of relatives of patients with myxoma. Chest 1994;105:65-8.

4. Viswanathan B, Luber JM, Bell-Thomson J. Right ventricular myxoma. Ann Thorac Surg 1985;39:280-1.

5. Heath D. Pathology of cardiac tumors. Am J Cardiol 1968;21: 315-27.

6. Burke AP, Virmani R. Cardiac myxoma: a clinicopathologic study. Am J Clin Pathol 1993;100:671-80.

\title{
CARDIAC TAMPONADE CAUSED BY PENETRATION OF AN ACUPUNCTURE NEEDLE INTO THE RIGHT VENTRICLE
}

\author{
Hajime Kataoka, MD, Oita, Japan
}

Acupuncture therapy, an ancient Chinese art, has been acknowledged throughout the world as a valid clinical procedure. In Japan, acupuncture needles are sometimes left embedded permanently in the musculature to relieve myalgia or neuralgia. ${ }^{1}$ In the case described here, traumatic cardiac tamponade was caused by an acupuncture needle, which had presumably migrated from a distant part of the body via the venous route and eventually penetrated the right ventricular free wall. X-ray computed tomographic imaging confirmed that cardiac tamponade had been caused by the needle.

Clinical summary. A 69 -year-old woman was admitted to Nishida Hospital with chest oppression, dyspnea, and cold sweating. At another clinic, she had been receiving acupuncture therapy (embedment of needles in the musculature) about one to three times per month for 10 years because of chronic upper and lower back pain. The latest acupuncture session had been conducted 2 months before the present admission to our hospital. The number of acupuncture needles (made of silver or stainless steel, each about $3 \mathrm{~cm}$ long and $0.05 \mathrm{~cm}$ in diameter) embedded in the musculature around the back of the neck to the shoulder and the lower back in each session ranged from one to five.

From the Department of Internal Medicine, Nishida Hospital, Oita, Japan.

Received for publication March 17, 1997; accepted for publication March 27, 1997.

Address for reprints: Hajime Kataoka, MD, Internal Medicine, Nishida Hospital, 3-3-24 Ohte machi, Saiki city, Oita 876, Japan.

J Thorac Cardiovasc Surg 1997;114:674-6

Copyright (C) 1997 by Mosby-Year Book, Inc.

$0022-5223 / 97 \$ 5.00+0 \quad \mathbf{1 2} / \mathbf{5 4} / \mathbf{8 2 3 5 3}$
On physical examination the patient had no fever and had pulsus paradoxus with a maximal systolic blood pressure of $80 \mathrm{~mm} \mathrm{Hg}$. Jugular venous distention and mild peripheral edema were present. The heart sounds were distant, but a friction rub was not audible. Chest and abdominal roentgenograms showed many embedded needles around the cervical and lumbar vertebrae. Several needles were superimposed on the lung field and the cardiac silhouette. The exact relationship between the needles and cardiac structures was not apparent on plain $\mathrm{x}$-ray films and fluoroscopic examination of the chest. Transthoracic echocardiography revealed massive pericardial effusion with early diastolic collapse of the right ventricle. Because of the patient's hemodynamic deterioration, a drainage tube was introduced into the pericardial space on an emergency basis, resulting in efflux of bloody effusion and prompt clinical improvement. The nature of the pericardial fluid suggested traumatic injury to the heart, and an acupuncture needle was considered to be the cause. Close echocardiographic reexamination including the transesophageal mode in addition to the transthoracic method failed to find any acupuncture needle in or around the cardiac structures. However, $\mathrm{x}$-ray computed tomographic imaging with contrast medium disclosed the metal-density material in the right ventricle, which stuck into the liver through the right ventricular free wall (Fig. $1, C$ to $F$ ). There were three needles in the pulmonary vasculature (one needle in the proximal right pulmonary artery is shown in Fig. 1, A).

One week after admission to my hospital, the patient was transferred to Oita Medical University and underwent cardiac surgery to remove the penetrating acupuncture needle. With cardiopulmonary bypass equipment available, the heart was exposed by a midsternal incision and the parietal pericardium was opened at the anterior 

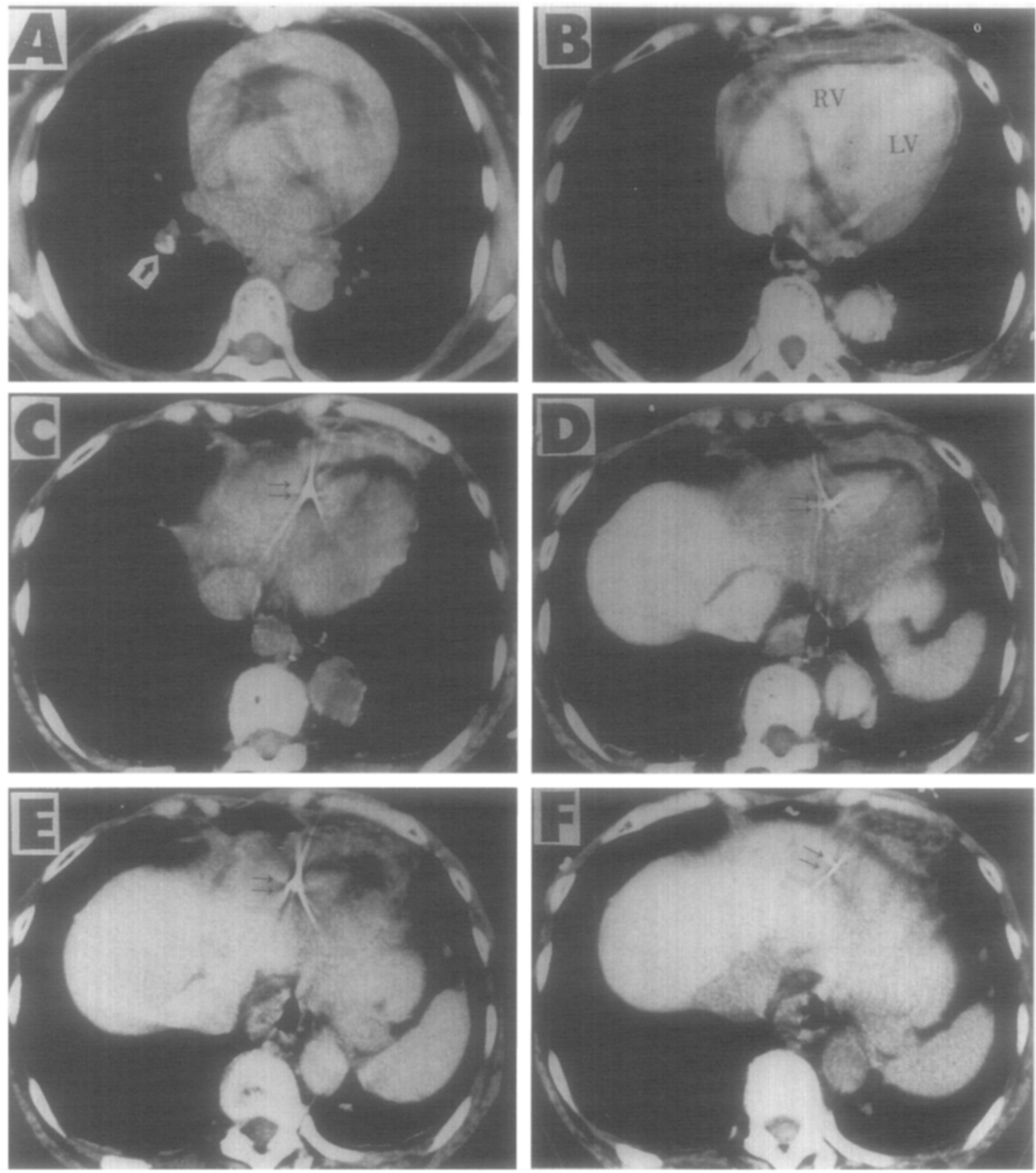

Fig. 1. Several horizontal slices of contrast $x$-ray computed tomographic images at the levels of proximal pulmonary artery $(\mathbf{A})$, lower half of the heart $(\mathbf{B}$ to $\mathbf{E})$ and upper portion of the liver $(\mathbf{F})$. Single arrow indicates metal-density material in the proximal pulmonary artery, and double arrows show metal-density material in the right ventricle, which extends into the upper portion of the liver. $L V$, Left ventricle; $R V$, right ventricle.

cardiac surface. About $50 \mathrm{ml}$ of semifluid blood had collected in the pericardial sac, but active bleeding was not observed. Aspiration of the pericardial bloody fluid disclosed a rust-free acupuncture needle, which had penetrated the inferior right ventricular free wall and stuck deeply into the diaphragmatic musculature. The needle was successfully withdrawn without the need for cardio- pulmonary bypass. The postoperative course was uneventful, and the patient has done well since hospital discharge.

Discussion. The heart or great vessels may be severely damaged by objects of unimpressive size or appearance. ${ }^{2}$ Examination of the literature shows that pins and needles often injure the heart, sometimes resulting in life-threatening cardiac tamponade. ${ }^{2}$ One of the most striking aspects of 
acupuncture therapy is the virtual absence of life-threatening side effects and complications resulting from its use. ${ }^{3}$ However, it would be expected that life-threatening cardiovascular complications like those in this report (i.e., cardiac tamponade) could result from the use of acupuncture needles. A previous report ${ }^{4}$ described a serious complication as a consequence of applying needles to the thorax directly, which caused cardiac injury and tamponade. Several pathways by which the acupuncture needle could have reached the heart can be considered, ${ }^{2}$ but we believe that in my patient the needle reached the right ventricle via the bloodstream from a distant region, eventually penetrating the free wall, because of the location of the acupuncture therapy and the coexistence of several needles in the pulmonary vasculature. In patients such as this, surgical removal of the pins or needles in the heart should be attempted because of the high mortality rate of needle-related cardiac injury. ${ }^{2}$ To facilitate successful removal of a needle from the heart, it is necessary to define the precise location of the needle before or during the operation. Ultrasonography has been reported to be useful for the evaluation and management of patients with foreign bodies in the heart, ${ }^{5}$ although I failed to find the cardiac needle before operating using this method. Instead, $\mathrm{x}$-ray computed tomography with contrast medium enabled me to locate the needle in the heart and greatly facilitated its successful removal, although the image was accompanied by an object-related artifact.
In conclusion, physicians involved in acupuncture therapy should keep in mind the possible serious cardiac complications reported here. Contrast x-ray computed tomography may be useful for imaging the needle in the heart and great vessels.

I thank Drs. Yoshiaki Mori and Tetsuo Hadama of the Department of Cardiovascular Surgery at the Oita Medical University for their help in treating the patient.

\section{REFERENCES}

1. Shiraishi S, Goto I, Kuroiwa Y, Nishio S, Kinoshita K. Spinal cord injury as a complication of acupuncture. Neurology 1979;29:1188-90.

2. Schechter DC, Gilbert L. Injuries of the heart and great vessels due to pins and needles. Thorax 1969;24:246-53.

3. Rapson LM. Acupuncture: a useful treatment modality. Acupunc Res Q 1984;8:15-27.

4. Rosted P. Literature survey of reported adverse effects associated with acupuncture treatment. Am J Acupunc 1996;24: $27-34$.

5. Harrison LH Jr, Kisslo JA Jr, Sabiston DC Jr. Extraction of intramyocardial foreign body utilizing operative ultrasonography. J Thorac Cardiovase Surg 1981;82:345-9.

\title{
LONG-TERM FOLLOW-UP OF APROTININ-SPECIFIC IMMUNOGLOBULIN G ANTIBODIES AFTER CARDIAC OPERATIONS
}

\author{
J. Weipert, MD, ${ }^{a}$ H. Meisner, MD, ${ }^{a}$ M. Jochum, $\mathrm{PhD},{ }^{b}$ and W. Dietrich, MD, ${ }^{c}$ Munich, Germany
}

The protease inhibitor aprotinin (Trasylol, Bayer AG, Leverkusen, Germany) is used in cardiac operations for acquired heart disease to reduce postoperative bleeding and allogeneic blood requirement. ${ }^{1}$ Aprotinin is a polybasic polypeptide derived from bovine lung, and antibody production in human beings is likely to occur. Recently,

From the Clinic of Heart and Vascular Surgery, German Heart

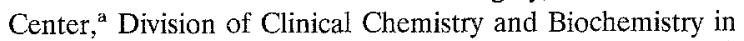
the Department of Surgery, Ludwig-Maximilians-University, ${ }^{b}$ and Institute of Anesthesiology, German Heart Center, Technical University of Munich, Munich, Germany.

Received for publication Dec. 23, 1996; accepted for publication March 12, 1997.

Address for reprints: Joachim Weipert, MD, Klinik für Herz- und Gefä $\beta$ chirurgie, Deutsches Herzzentrum München, Lazarettstr. 36, 80636 München, Germany.

J Thorac Cardiovasc Surg 1997;114:676-8

Copyright $\mathbb{0} 1997$ by Mosby-Year Book, Inc.

$0022-5223 / 97 \$ 5.00+0 \quad \mathbf{1 2 / 5 4 / 8 1 9 4 8}$ deaths resulting from anaphylactic reactions after systemic aprotinin exposure in cardiac operations have been reported. ${ }^{2}$ However, it is unknown by which pathway these reactions are mediated. Because the use of aprotinin at initial cardiac operations is widespread, at least in Europe, the risk for anaphylactic reactions at aprotinin reexposure may be increased. To study parameters to assess patients' individual risks for adverse reactions to aprotinin, we followed the course of aprotinin-specific antibodies of the immunoglobulin (IG) $G$ class for 4 years.

With institutional approval and after informed consent, 36 consecutive adult patients having cardiac operations could be followed up. Only patients with no previous exposure to aprotinin were selected for this study. Blood specimens were drawn for the first time during their postoperative hospital stay or on an outpatient basis, but within 4 months after the operation. Four years later these patients were reinvestigated. Aprotinin was used according to the following protocol: A test dose of $10,000 \mathrm{KIU}$ of aprotinin $(1 \mathrm{ml})$ was given 10 minutes before the first bolus. Patients received an initial bolus of $2 \times 10^{6} \mathrm{KIU}$ of 